\title{
Evolution of the $\beta$ Phase Flux Dissolution during Quasicrystal Formation
}

\author{
M. SurowiEC ${ }^{a, *}$, W. BOGDANOWICZ ${ }^{a}$ AND M. SOZAŃSKA ${ }^{b}$ \\ ${ }^{a}$ University of Silesia, Institute of Material Science, 41-500 Chorzów, Poland \\ ${ }^{b}$ Silesian University of Technology, Faculty of Materials Engineering and Metallurgy, 40-019 Katowice, Poland \\ This study focuses mainly on the verification of a peritectic reaction during the crystallization of quasicrystals. \\ The $\beta$ and $\lambda$ phases apparently provide the necessary material for crystallization of the quasicrystalline $\psi$ phase. \\ We present results of an experimental visualization of the evolution of the flux dissolution process of the high \\ temperature $\beta$ phase. Solidified droplets of the $\beta$ phase, formed primarily by the surface tension of the liquid \\ within a bubble or a shrinkage, after crumbling during the flux dissolution may contribute to the quasicrystal \\ formation process according to a peritectic reaction: $\beta_{\mathrm{c}}+\lambda+L \rightarrow \psi$, where $\beta_{\mathrm{c}}$ denotes the crumbled $\beta$ phase \\ immersed in the liquid.
}

DOI: 10.12693/APhysPolA.126.594

PACS: $81.10 . \mathrm{Fq}, 61.44 . \mathrm{Br}$

\section{Introduction}

According to a common view, icosahedral as well as decagonal quasicrystals form within a peritectic reaction between the liquid phase and certain high temperature phases. Below the peritectic temperature the quasi-crystal coexists with the liquid phase.

In the case of icosahedral quasicrystals of the $\mathrm{Al}-\mathrm{Cu}-\mathrm{Fe}$ system two high temperature phases take part in the reaction, namely a cubic $\beta$ phase and a monoclinic $\lambda$ phase [1-3]. Additionally, as a result of the peritectic transition, a tetragonal $\omega$ phase is formed in the system [4]; it was observed in a single crystal form [5]. Peritectic phase transformations were found to have a common feature major consumption of the CsCl-type cubic $\beta$ phase [6]. However, the details of this "consumption" have not yet been explained.

Ishimasa and Mori [7] noted that quasicrystalline grain growth seemed to be associated with the formation of liquid during the annealing treatment above the peritectic temperature. A grain growth was not observed when no annealing at a lower temperature took place, suggesting that both the formation of a liquid and annealing below the peritectic temperature was necessary for the growth of the $\psi$ phase grains.

Lograsso et al. [8] also stressed that both the macrostructure and microstructure of the as-grown $\psi$ phase grains provide an indirect evidence of the presence of a liquid at the growth temperature as the fivefold faceting of grains must result from contact with a liquid phase. In their opinion, the liquid forms at the growth temperature as a result of passing from the single-phase $\psi$ field into one of two phases $(\psi+$ liquid $)$.

This paper deals with the subsequent stages of flux dissolution morphology of a high temperature single crys-

*corresponding author; e-mail: marian.surowiec@us.edu.pl talline cubic $\beta$ phase. An evident gradual flux dissolution process of the $\beta$ phase provides material for formation of the quasicrystalline $\psi$ phase. The crystallization process of the $\psi$ phase is discussed in view of a peritectic reaction of the crumbled $\beta$ phase immersed in the liquid. The reaction is apparently different from a classic peritectic reaction since the quasicrystals have facetted equilibrium shapes.

\section{Experimental details}

Equilibrium growth forms of the $\beta$ phase were formed in ingots of an $\mathrm{Al}-\mathrm{Cu}-\mathrm{Fe}$ alloy, for which the nominal composition was 65 at.\% Al, 20 at.\% $\mathrm{Cu}$ and 15 at.\% Fe. The ingots of total mass of approximately $40 \mathrm{~g}, 15 \mathrm{~mm}$ in diameter and $60 \mathrm{~mm}$ high, were sharpened at the bottom. The specimens were synthesized in a helium atmosphere inside alumina crucibles starting from $99.997 \%$ pure $\mathrm{Al}, 99.9 \%$ pure $\mathrm{Cu}$ and $99.98 \% \mathrm{Fe}$ using the Bridgman-Czochralski growth (BCG) apparatus equipped with an induction furnace. The initial temperature of the melt was increased up to $1550^{\circ} \mathrm{C}$ and the crystallization rate during the Bridgman directional crystallization was $6 \mathrm{~mm} / \mathrm{h}$ resulting in an approximate cooling rate of $1.3^{\circ} \mathrm{C} / \mathrm{min}$. Single crystal grains have crystallized in the melt or were subject to the flux dissolution until the melt has been removed by the solidification shrinkage. The ingot was crushed into several pieces and different growth forms previously closed inside cavities and pores were subject to the investigation of growth morphology by the scanning electron microscopy (SEM) using primary and secondary electrons. The selected area diffraction (SAD) as well as the X-ray powder diffraction confirmed the phase identification of the predicted phase composition in the $\mathrm{Al}-\mathrm{Cu}-\mathrm{Fe}$ system.

\section{Results}

After crushing the ingots into several pieces various growth forms previously closed inside cavities and pores 
were observed by the SEM. Droplets of the $\beta$ phase formed primarily by the surface tension of liquid within a bubble or shrinkage hollow (cavity) solidified in their initial liquid shape (Fig. 1a-c). Facetted single quasicrystals of the $\psi$ phase are observed in the vicinity of single crystalline $\beta$ phase droplets (Fig. 1d).

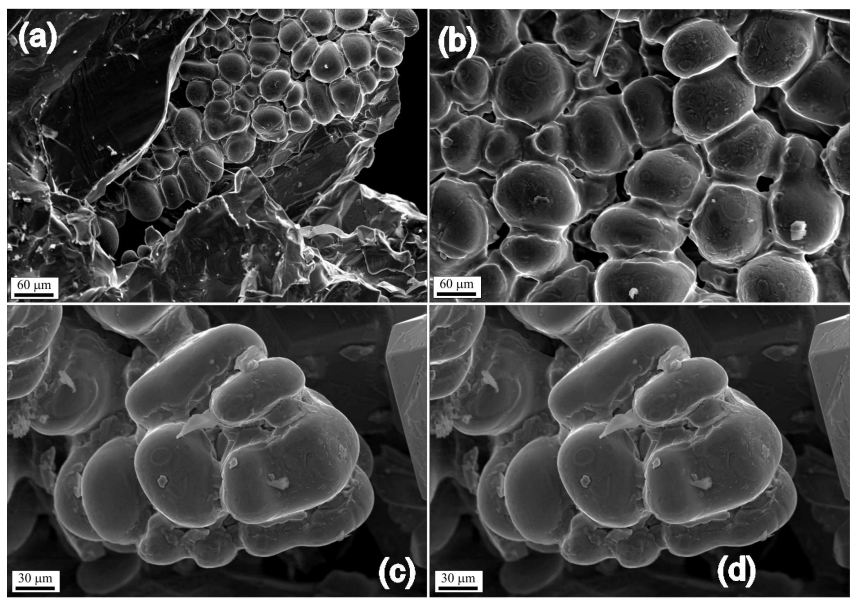

Fig. 1. Droplet-like morphology of the high temperature $\beta$ phase revealed inside shrinkage cavities of the ingot $(\mathrm{a}, \mathrm{b}, \mathrm{c})$ and polyhedral single quasicrystals ( $\psi$ phase) emerging from the melt (d).

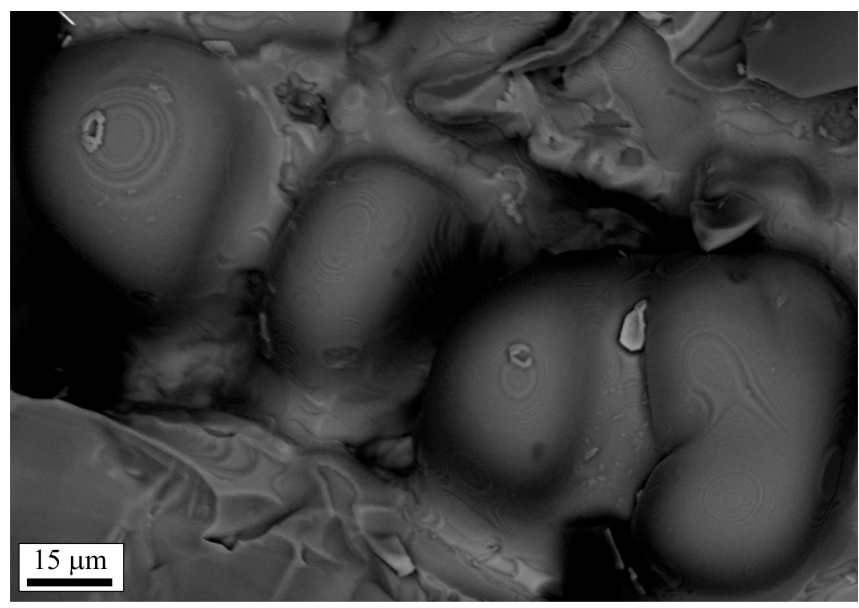

Fig. 2. Initial stage of the flux dissolution of the high temperature $\beta$ phase.

The first stage of the flux dissolution process of the $\beta$ phase reveals tiny concentric circular steps visible on single crystalline droplets (Fig. 2).

In an advanced stage of the flux dissolution process of the $\beta$ phase more concentric rings as well as more steps develop (Fig. 3). The arrangement of steps within one set of concentric rings reveals the local symmetry of a droplet as the rings transform into polygons - hexagons or octagons revealing threefold or fourfold symmetry axes, respectively (Fig. 4). Also a twofold symmetry axis can

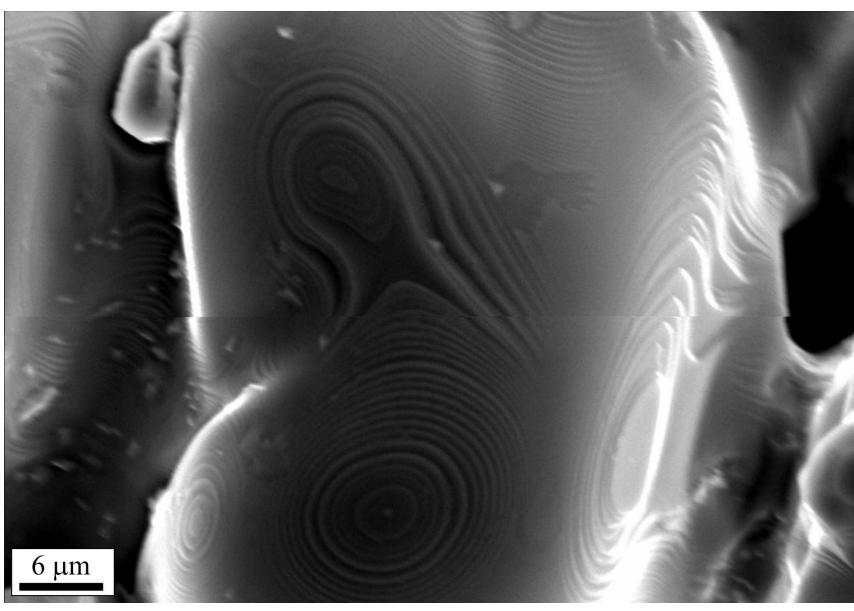

Fig. 3. Morphology of the $\beta$ phase droplets after an advanced flux dissolution process.

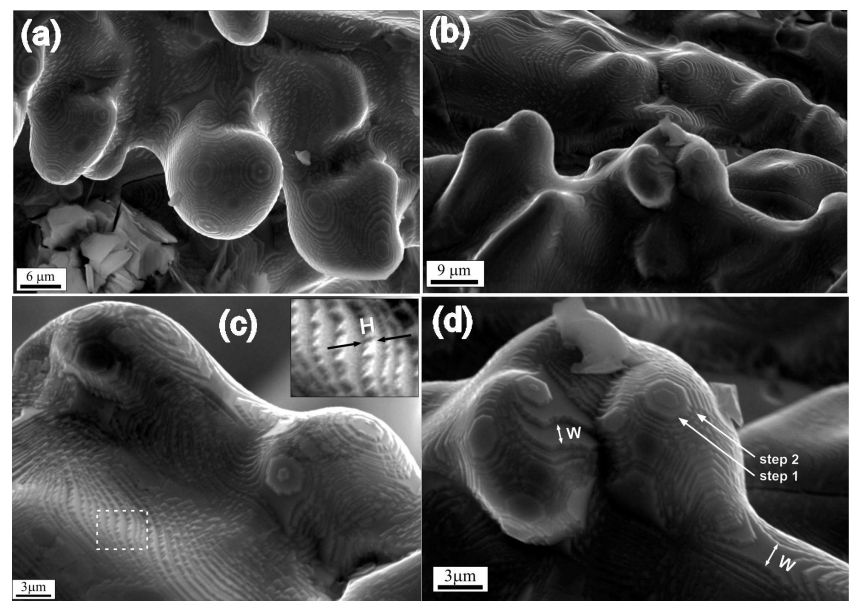

Fig. 4. Final observed stages of the flux dissolution of the single crystalline $\beta$ phase; $(\mathrm{a}, \mathrm{b})$ terraces visible on solidified droplets and $(\mathrm{c}, \mathrm{d})$ terraces revealed on a waved surface.

be found. The observed unique long terraces are formed by low index crystallographic planes like (100), (110), or (111). The width $(W)$ of terraces is up to $2.5 \mu$ m whereas well defined terrace steps are about $0.1 \mu \mathrm{m}$ up to $0.3 \mu \mathrm{m}$ high and their height $(H)$ also differs (Fig. 4c,d). The surface of terrace steps is irregular, waved and usually inclined to the flat terraces. The dissolution process apparently is realized by removing small fragments of steps resulting in a wavy surface of the steps. The contrast between the terrace width and its height results from the dissolution rate, which is higher along the direction perpendicular to the terrace step (Fig. 4c).

\section{Conclusion}

Our experimental results show a gradual dissolution process (rather than contamination) of the $\beta$ phase. This plays a crucial role during subsequent crystallization. 
Facetted equilibrium shapes of quasicrystals are formed by a peritectic reaction. Well defined steps of terraces approximately $0.2 \mu \mathrm{m}$ high revealed in the final stage of dissolution show that the dissolution process does not occur atom by atom. The liquid is continuously enriched by a permanent dissolution of the $\beta$ phase.

These observations are not sufficient to describe in detail a formation mechanism for equilibrium shapes of quasicrystals within a peritectic reaction. However, crumbled fragments of $\beta$ phase may catalyse a classic peritectic reaction (between the melt and the $\beta$ phase) producing quasicrystalline ledges. Gastaldi et al. [9] during a synchrotron in situ observation concluded that the process of facetted growth of $\mathrm{Al}-\mathrm{Mn}-\mathrm{Pd}$ quasicrystal is proceeding by a lateral motion of ledges at the solid-melt interface and is controlled by the interface kinetics.

Quasicrystals exhibiting facetted equilibrium shapes are apparently formed by a modified peritectic reaction $\beta_{\mathrm{c}}+\lambda+L \rightarrow \psi$, where $\beta_{\mathrm{c}}$ denotes the crumbled $\beta$ phase immersed in the liquid. On a macro scale the crystallization process is analogous to the flux technique of crystal growth resulting in facetted single crystals.

The crystallisation process including the peritectic reaction has a local character as the observed stable polyhedral growth forms can be explained by the ScheilGulliver approach to a non-equilibrium solidification. This approach approximates a non-equilibrium solidification by assuming a local equilibrium of the advancing solidification front at the solid-liquid interface [10].

\section{References}

[1] F.W. Gayle, A.J. Shapiro, F.S. Biancanello, W.J. Böttinger, Metall. Trans. A 23, 2409 (1992).

[2] D. Gratias, Y. Calvayrac, J. Devaud-Rzepski, F. Faudot, M. Harmelin, A. Quivy, P.A. Bancel, J. NonCryst. Solids 153-154, 482 (1993).

[3] T.A. Lograsso, D.W. Delaney, J. Mater. Res. 11, 2125 (1996).

[4] F. Faudot, A. Quivy, Y. Calvayrac, D. Gratias, M. Harmelin, Mater. Sci. Eng. A 133, 383 (1991).

[5] M. Surowiec, W. Bogdanowicz, J. Krawczyk, B. Formanek, M. Sozanska, Philos. Mag. 91, 2458 (2011).

[6] L.M. Zhang, J. Schneider, R. Lück, Intermetallics 13, 1195 (2005).

[7] T. Ishimasa, M. Mori, Philos. Mag. Lett. 62, 357 (1990).

[8] T.A. Lograsso, A.R. Ross, D.W. Delaney, MRS Proc. 553, 3 (1998); Processing of Al-Cu-Fe Single Grains (1998), Ames Laboratory Conference Papers, Posters, and Presentations. Paper 36, (1998).

[9] J. Gastaldi, G. Reinhart, H. Nguyen-Thi, N. Mangelinck-Noel, B. Billia, T. Schenk, J. Hartwig, B. Grushko, H. Klein, A. Buffet, J. Baruchel, H. Jung, P. Pino, B. Przepiórzyński, Philos. Mag. 87, 3079 (2007).

[10] E. Scheil, Z. Metallk. 34, 70 (1942). 